\title{
CONSTRUYENDO LA BARCELONA CREATIVA: NUEVOS ACTORES, NUEVAS ESTRATEGIAS
}

\author{
Montserrat Pareja-Eastaway ${ }^{1}$
}

\begin{abstract}
Resumen - Barcelona está en el punto de mira internacional. La ciudad que triunfó con la organización de los Juegos Olímpicos en el año 1992 quiere ser hoy una ciudad creativa del siglo XXI. Para ello hace falta propiciar las condiciones que contribuyan a generar un discurso compartido sobre la capacidad de Barcelona para convertirse en una ciudad atractiva para el talento creador y también para las empresas. Tanto el reconocimiento del sustrato histórico-económico inicial como punto de partida hacia la ciudad creativa, junto con el papel motor de la cultura en la generación de un peculiar y distintivo bagaje hacen de Barcelona una ciudad diferente. Sin embargo, esto no es suficiente. La participación de todos los actores alrededor de una estrategia compartida se convierte en Barcelona en el mayor reto de futuro así como la minimización de los efectos negativos que inevitablemente acompañan al éxito. Empresas, instituciones y ciudadanía constituyen el mejor activo de la ciudad: es necesario aunar esfuerzos y aprovechar los efectos que se generan entre ellos. El consenso y la participación van más allá de meras utopías, siendo imprescindibles para la ciudad del mañana. La iniciativa pública ejerce el liderazgo en Barcelona: el surgimiento de puentes conectores entre necesidades de los sectores creativos con los mecanismos de intervención pública es, sin lugar a dudas, la mejor garantía de éxito.
\end{abstract}

Palabras clave: Barcelona, ciudad creativa, trayectorias urbanas, liderazgo público, factores "hard" y "soft", "network" de localización.

Resumo - CONSTRUINDO A BARCELONA CRIATIVA: NOVOS ACTORES, NOVAS ESTRATÉGIAS. Barcelona está na mira internacional. A cidade que triunfou com a organização dos Jogos Olímpicos em 1992 procura no século XXI afirmar-se como uma cidade criativa. Mas é necessário criar as condições para que Barcelona se converta numa cidade atractiva para o talento criativo e as empresas. O reconhecimento do substrato histórico-económico, como ponto de partida para a cidade criativa, juntamente com o papel motor da cultura na geração de uma bagagem distintiva e peculiar, fazem de Barcelona uma cidade diferente. No entanto, isto não é suficiente. A participação de

Recebido: 15/06/2010. Aceite: 29/10/2010.

1 Professora da Universidad de Barcelona GRC Creatividad, Innovación y Transformación Urbana, Facultad de Economía y Empresa. E-mail: mpareja@ub.edu 
todos os actores em torno de uma estratégia partilhada coloca a Barcelona o maior desafio do futuro, assim como a minimização dos efeitos negativos que inevitavelmente acompanham o êxito desta estratégia. Empresas, instituições, cidadania constituem os melhores activos da cidade: é necessário unir esforços e aproveitar os efeitos que se geram entre eles. O consenso e a participação vão além de meras utopias convertendo-se em factores imprescindíveis na cidade de amanhã. A iniciativa pública exerce liderança em Barcelona: as conexões entre as necessidades dos sectores criativos e os mecanismos de intervenção pública são, sem dúvida, a maior garantia de sucesso.

Palavras-chave: Barcelona, cidade criativa, trajectórias urbanas, liderança pública, factores "hard"e "soft", "network" de localização.

\begin{abstract}
Building A Creative Barcelona: neW aCtors, NeW StRategies. Barcelona is under the international spotlight. The city that triumphed with the organisation of the Olympic Games in 1992 now wants to become a $21^{\text {st }}$ Century creative city. In order to achieve this goal, the city must establish conditions that facilitate the emergence of a shared discourse around its ability to become an attractive city for creative talent and for businesses. The recognition of the historical-economic heritage, as a starting point for the creative city, along with the driving role played by culture in the generation of a exclusive and distinctive experience, turn Barcelona into a unique city. However, this is not sufficient. The participation and involvement of all the actors in a shared strategy pose significant challenges to Barcelona, as does the need to minimise the negative effects that inevitably accompany success. Companies, institutions and citizens constitute the city's best assets: they must work in partnership and take advantage of the synergies generated amongst them. Consensus and participation are more than mere utopias in Barcelona: they have become requirements for the city of tomorrow. Moreover, leadership in Barcelona is largely left to public initiative: the emergence of linkages across the needs of the various creative sectors, based on public intervention mechanisms is the best way to ensure success.
\end{abstract}

Keywords: Barcelona; creative city; urban pathways; public leadership; "hard", "soft" and "network" localisation factors.

Résumé - La CONSTRUCTION D’une BARCELONE CRÉATIVE: NOUVEAUX ACTEURS ET NOUVELLES STRATÉGIES. Barcelone a atteint une position internationale. La ville qui a organisé triomphalement les Jeux Olympiques en 1992 veut désormais être une des villes créatrices du $\mathrm{XXI}^{\mathrm{e}}$ siècle. Mais manquent pour cela les conditions permettant de créer un discours généralement accepté sur la capacité qu'aurait Barcelone à devenir une ville attractive pour les talents créateurs et les entreprises. Son originalité vient tant de son substrat historique et économique initial que du rôle moteur qu'y joue une culture très originale. Mais ce n'est pas suffisant. Il faut que les divers acteurs s'unissent dans une stratégie commune et que les aspects négatifs qui accompagnent forcément les succès soient minimisés. Les entreprises, les institutions et la citoyenneté sont les meilleurs atouts de la ville : ils doivent joindre leurs efforts pour mieux réussir. A Barcelone, l'entente et la participation ne sont pas des utopies, mais des nécessités, indispensables pour créer la ville de demain. L'initiative publique y étant dominante, une bonne liaison entre les besoins des secteurs créatifs et les mécanismes d'intervention publique sera la meilleure garantie du succès. 
Mots clés: Barcelone, ville créative, trajectoires urbaines, leadership publique, facteurs «hard» et «soft», «network» de localisation.

\section{INTRODUCCIÓN}

La ciudad de los prodigios, la ciudad de las mil caras, la ciudad de los arquitectos... estos son, entre otros, apelativos que la literatura ha otorgado a Barcelona. Esta ciudad, con una permanente preocupación por encontrarse a sí misma y definir epítetos que la resuman, es hoy considerada como una de las ciudades europeas más atractivas para vivir, especialmente por parte de los colectivos que trabajan en actividades vinculadas a la creatividad y el conocimiento (Pareja-Eastaway et al., 2009). No es casualidad que tanto desde las instancias municipales como desde otros organismos que surgen de la sociedad civil (i.e. Plan Estratégico Metropolitano de Barcelona) se quiera convertir a Barcelona en una ciudad con una elevada especialización económica basada en la creatividad y el conocimiento.

Barcelona ha sido capaz de formular recetas convincentes de éxito y seguir paso a paso el camino diseñado para ello. Un buen ejemplo fue la celebración de los Juegos Olímpicos en el año 1992. Desde entonces, la búsqueda de nuevas fórmulas ha sido recurrente, conllevando mayores o menores éxitos en su puesta en escena. Actualmente, Barcelona persigue sumarse al privilegiado grupo de ciudades en la esfera internacional que dicen llamarse 'creativas'.

Este artículo se plantea identificar cuáles son los condicionantes que han provocado la consideración de Barcelona como una ciudad creativa. Para ello, la primera sección resume brevemente las principales aportaciones teóricas entorno a esta temática para, a continuación, a la luz de los argumentos claves definidos, contrastar estas hipótesis con la realidad de Barcelona. En particular, se hará hincapié en los elementos clave que permiten, tanto a través del legado de la historia como de acuerdo a las estratégicas programáticas de la gobernanza de la ciudad, convertir hoy a Barcelona en una ciudad creativa.

\section{LA CIUDAD CREATIVA, UNA TENDENCIA DEL SIGLO XXI}

Los cambios en los modos de producción económica han determinado la emergencia de diferentes tipologías de ciudades. Desde la aglomeración cercana a las fuentes energéticas hasta la urbe tecnológica, las ciudades son el reflejo de los cambios acontecidos en la esfera económica que, a su vez, han influido tanto en las relaciones sociales como en las formas institucionales.

La literatura académica en sus diferentes disciplinas es un terreno fértil para el debate en el que la sistematización y la síntesis muestra aquello que se entiende por ciudad en cada momento: la ciudad post-fordista (Amin, 1994), la ciudad global o globalizada (Sassen, 1991; Marcuse y van Kempen, 2000) y la 
ciudad digital (Aurigi, 2005) son conocidos ejemplos que vindican los principales atributos de estas manifestaciones territoriales de las dinámicas en los modos de producción. La Nueva Economía va ligada a nuevos modelos de ciudad: actualmente, la búsqueda del éxito en la creación de riqueza y la mejora de la competitividad urbana, representan los principales objetivos que persiguen las ciudades, especialmente en el mundo desarrollado. De este modo, la denominada 'tercera revolución industrial' plantea nuevas apuestas competitivas en las que el talento surge como eje vertebrador. Estos elementos adquieren un peculiar significado ante la madurez de las tendencias globalizadoras iniciadas hace décadas. El crecimiento de las interdependencias entre países, la mejora en los flujos de conectividad y la extensión de las infraestructuras de transporte ha cambiado los parámetros relacionales de las ciudades. El siglo XXI muestra un nuevo panorama de ciudades creativas, en dónde el conocimiento y el capital humano juegan un factor esencial para el futuro de la ciudad.

Sin embargo, la ciudad creativa en términos conceptuales surge a finales de los años 80 más como aspiración que como respuesta a los problemas emergentes (Landry, 2000). La creatividad no permite su apropiación por ningún colectivo o sector sino que está en manos de todos aquellos que se enfrentan ante dilemas y oportunidades. La creatividad consiste en realidad, en adoptar un nuevo enfoque resolutivo ante los problemas, desde los más cotidianos a los más puntuales, tanto en esferas privadas como públicas. De este modo, la ciudad creativa aúna la búsqueda de nuevas soluciones por diferentes actores en escenarios diversos. En este contexto, libros como el de Florida (2002) se popularizan a escala mundial debido, entre otros, a su particular análisis del concepto de 'clase' en este nuevo marco de referencia. Incluso en el supuesto de contradecir la línea argumental presentada por este autor, existe coincidencia en el debate académico acerca del cambio estructural sufrido por los protagonistas del crecimiento económico; hoy, el trabajador creativo, con talento y alta cualificación, emerge como pieza clave en la transformación económica.

Si el talento y la creatividad son elementos fundamentales en los nuevos retos del mundo globalizado, la pregunta inmediata es: ¿qué papel resta a las ciudades? Tal y como señala Inneraritty (2007), los individuos ya no pertenecen a una única comunidad, su vida se reparte entre diferentes redes sin garantía de exclusividad. De ahí que el territorio no desaparece pero sí se transforma la relación del individuo con él: "Todo el espacio se ha vuelto simbólico, lo que permite configuraciones más flexibles que en la época en que los espacios estaban bajo la pretensión de monopolio exclusivo. Un espacio relacional y múltiple posibilita unos compromisos más abiertos que cuando el territorio era una dimensión fija, objetiva y rígida en la vida de los hombres y las sociedades." (Inneraritty, 2007: 5)

Ciertamente, los ejes de la comunicación social y profesional en red determinan el papel reservado para la ciudad, ahora convertida en polo de atracción de actividad creativa y de alto valor añadido. Aunque las industrias culturales o creativas surgen como hard core de la nueva ciudad, la transformación del enfo- 
que se expande a toda la dimensión del sistema productivo, existiendo algunas condiciones que facilitan la nueva configuración de las ciudades. Tal y como Landry (2000) señala, curiosidad, imaginación, creatividad, innovación e invención son los cinco elementos básicos que favorecen la capacidad de la ciudad y de su tejido social y productivo para proveer de nuevas soluciones, tanto a problemas tradicionales como emergentes.

Ciertos elementos parecen favorecer el desarrollo de las industrias culturales o creativas y, simultáneamente, el posicionamiento de la ciudad como 'creativa' en el contexto internacional. A pesar de las aparentes diferencias entre autores, no existe un debate de fondo acerca de las condiciones o pre-requisitos que favorecen la aparición de ciudades creativas (fig. 1).

\begin{tabular}{|l|}
\hline \multicolumn{1}{|c|}{$\begin{array}{c}\text { Törnqvist (1983) } \\
\text { Aspectos clave }\end{array}$} \\
\hline $\begin{array}{l}\text { Información transmitida } \\
\text { entre personas } \\
\text { Conocimiento basado en la } \\
\text { acumulación de información } \\
\text { Competencia en ciertas } \\
\text { actividades } \\
\text { Creatividad (algo nuevo en } \\
\text { base a lo anterior) }\end{array}$ \\
\end{tabular}

\begin{tabular}{|l|}
\hline \multicolumn{1}{|c|}{$\begin{array}{c}\text { Andersson (1985) } \\
\text { Pre-requisitos }\end{array}$} \\
\hline $\begin{array}{l}\text { Conocimiento original y altos } \\
\text { niveles de competencias } \\
\text { Desequilibrio entre la } \\
\text { necesidad de experiencia y } \\
\text { oportunidades actuales } \\
\text { Un milieu diverso } \\
\text { Buenas posibilidades para } \\
\text { transporte y comunicación } \\
\text { Una inestabilidad estructural } \\
\text { que facilite el desarrollo } \\
\text { sinérgico }\end{array}$ \\
\hline
\end{tabular}

\begin{tabular}{|l|}
\hline \multicolumn{1}{|c|}{$\begin{array}{c}\text { Malecki (1987) } \\
\text { Síntesis }\end{array}$} \\
\hline $\begin{array}{l}\text { La presencia de empleo } \\
\text { profesional cualificado } \\
\text { La aglomeración urbana en } \\
\text { dónde tiene presencia la } \\
\text { actividad cultural y la } \\
\text { comunicación } \\
\text { Condiciones para promover la } \\
\text { sinergia o la inestabilidad }\end{array}$ \\
\end{tabular}

Fig. 1 - Tres aproximaciones a los condicionantes para ser una ciudad creativa.

Fig. 1 - Three approaches to the requirements for becoming a creative city.

A modo de síntesis, y siguiendo a Landry (2000), dos grandes conjuntos de factores, personales y colectivos, favorecen las condiciones previas para que una ciudad sea 'creativa'. De entre ellos distingue los siguientes: Cualidades personales, voluntad y liderazgo, diversidad humana y acceso al talento variado, cultura organizativa, identidad local, espacios urbanos y equipamientos $\mathrm{y}$, finalmente, dinámicas de red. Siguiendo esta argumentación, los factores clásicos de localización de las industrias en las ciudades - hard factors - (i.e. buenas infraestructuras, precio del suelo asequible, etc.) no funcionarían exactamente igual para las industrias creativas siendo más relevante la existencia de otro tipo de factores - soft factors - (i.e calidad residencial, atmósfera, tolerancia, etc.). Nuevas aportaciones (Musterd and Murie, 2010) al debate acerca de qué contribuye a la ubicación de la economía creativa en una ciudad señalan como elementos de peso los factores relacionales - network factors - (i.e. haber nacido en la ciudad, contactos sociales y profesionales, etc.). 
Además, otros autores (Kóvacs, et al., 2007) señalan la importancia de la contextualización de una determinada ciudad en su propia trayectoria histórica - pathways - contemplando globalmente aspectos económicos, culturales $\mathrm{y}$ políticos. Ello contribuye decisivamente a la unicidad de cada una de las ciudades que hoy dicen llamarse 'creativas'. En síntesis, dos dimensiones son excepcionalmente importantes para la ciudad competitiva en la economía moderna, la económica y la social. De hecho, un buen aprovechamiento de la tradición y la experiencia junto con el dinamismo propio de la aplicación de la innovación y la creatividad favorece la implantación de nuevos procesos productivos competitivos en la esfera internacional. Así, las ciudades que saben aprovechar su trayectoria histórica maximizan la probabilidad de situarse en los mejores puestos de la jerarquía urbana. De hecho, Scott (2006) señala la corriente que llega a finales de los años 80 y sensibiliza la conciencia de muchos estrategas políticos acerca de las posibilidades de 'vender' aquellos recursos existentes (más o menos latentes) en cuanto a bagaje histórico-artístico y patrimonio cultural. La explotación de estos activos pasa a ser un elemento clave en la estrategia competitiva de muchas de las ciudades, no tanto por su aportación a la cadena de valor como por su contribución al marketing de una determinada imagen de ciudad a escala internacional.

\section{BARCELONA HOY: UNA CIUDAD ATRACTIVA A ESCALA INTERNACIONAL}

Barcelona, al igual que Ámsterdam u otras ciudades europeas, es un ejemplo de ciudad que, en la actualidad, se incluye generalmente en la posición sub-top del ranking urbano internacional. Barcelona emerge en este contexto debido a múltiples razones, algunas de ellas intrínsecamente asociadas a la capacidad de la ciudad como organizadora de eventos a escala internacional, otras vinculadas a su deseo explícito de convertirse en la sede de numerosas sedes de empresas multinacionales. Otras argumentaciones que se aducen para explicar este relativo repentino estrellato en el panorama de ciudades responden a los esfuerzos en publicitar la ciudad en todos y cada uno de los aspectos en los que resulta competitiva, la creación de la 'marca' Barcelona y su exportación exitosa al ámbito internacional no es pues ajeno a este proceso. Además, la ciudad ofrece, a diferencia de otras ciudades españolas e incluso europeas, ciertos aspectos vinculados a la calidad de vida, a la multiculturalidad y a la tolerancia.

Desde los años 80 hasta la actualidad, Barcelona se ha caracterizado por su voluntad de internacionalización a través de la organización de numerosos eventos con repercusión global, la organización de ferias y congresos de alcance mundial $^{2}$ y el establecimiento de años temáticos estrechamente ligados al

2 Festival Sònar, Bread and Butter, The Brandery y Salón del Automóvil, entre otros. 
conocimiento, la cultura y la creatividad ${ }^{3}$. Este empeño no es independiente de un modelo particular de desarrollo urbano que aprovecha las oportunidades para reformular urbanísticamente algunas zonas de la ciudad (Clusa, 1999).

La mejora de la imagen de competitividad económica de Barcelona es incuestionable. Ciertos indicadores lo constatan: así, el European Cities Monitor (2009) señala la ciudad entre las cinco primeras que ofrecen oportunidades de negocios en Europa (solamente ultrapasada por Londres, París, Frankfurt y Bruselas) y la primera en términos de calidad de vida (siendo este el quinto año consecutivo liderando esta posición). La ciudad se encuentra también entre las diez más visitadas de Europa. Éste último indicador favorece las cotas de popularidad de la ciudad, visible tanto en la atracción de turismo (Barcelona ha consolidado su posición como uno de los principales destinos de turismo urbano en Europa, siendo el puerto mediterráneo líder en cruceros) como en su favorable posición para la ubicación de talento creativo.

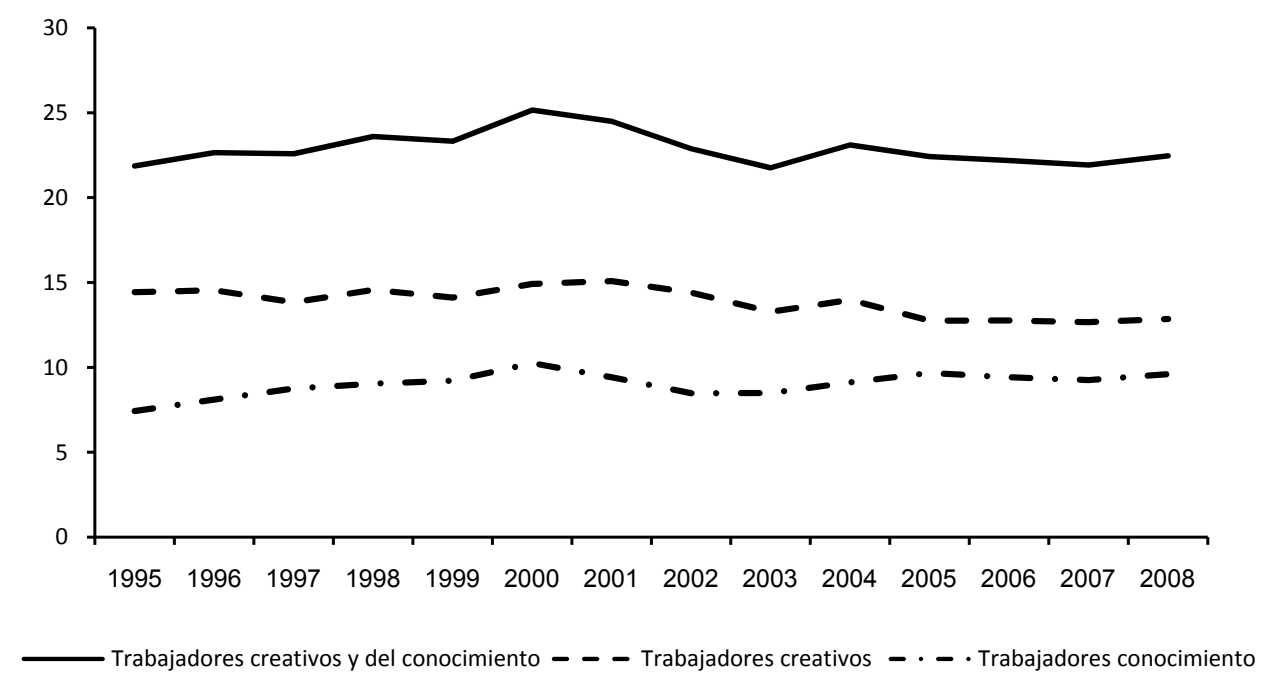

Fuente: INE(+): Los sectores han sido seleccionados de acuerdo a la metodología utilizada en el proyecto ACRE. (*) Sectores creativos: Publicidad, arquitectura, arte, antigüedades, artesanías, diseño, diseño de moda, video, cine, música, fotografia, artes visuales, edición, juegos de ordenador, publicaciones electrónicas, software, radio y televisión (**) Sectores del conocimiento: Servicios a las empresas (auditoria, contabilidad, etc.), sector financiero, $R+D$, TICs, educación superior.

Fig. 2 - Trabajadores en sectores creativos y en sectores del conocimiento sobre total empleo (+). Barcelona provincia (\%).

Fig. 2 - Share of creative and knowledge workers in total employment. Barcelona province (\%).

3 Año Gaudí (2005), Año Internacional del Libro (2006), Año Internacional de la Ciencia (2007), Año Cerdà (2009). 
El papel de las actividades creativas y, en particular, de las industrias culturales, resulta de gran relevancia para la ciudad tanto en términos de su contribución al empleo (fig. 2) como por su capacidad multiplicadora de atracción de otras actividades relacionadas. De este modo, de acuerdo a la metodología usada por el proyecto ACRE (Accommodating Creative Knowledge - Competitiveness of European Metropolitan Regions in an Enlarged Union), Barcelona se sitúa en tercer lugar en cuanto a la aportación al empleo de los sectores creativos (12\% sobre el total), después de Milán (14\%) y Budapest (13\%).

La ciudad de Barcelona ha aprovechado a oportunidad presentada por la crisis económica para afianzar el nuevo contenido de los principales motores del crecimiento económico de la ciudad ${ }^{4}$. Ello no es ajeno a una voluntad política decidida que ha apostado durante años por un cambio de modelo económico: "la ciudad ha seguido desarrollando una estrategia económica subyacente con una estructura ya en marcha desde hace años, promocionando un modelo de crecimiento económico basado en el conocimiento, la creatividad y la innovación, que conlleva un posicionamiento de liderazgo ante la Unión Europea como uno de los 'centros del conocimiento' del continente" (Observatori Barcelona, 2008: 1).

\section{EL SUSTRATO CREATIVO DE BARCELONA: LA IMPORTANCIA DEL CONTEXTO}

Tal y como se ha mencionado anteriormente, Barcelona aparece en el panorama internacional de forma reciente, nutriéndose de los frutos sembrados durante la celebración olímpica y aprovechando la generación de efectos externos consecuencia del cambio de valores provocado por las nuevas formas de producción basadas en el conocimiento y la creatividad. Barcelona ofrece el ambiente ideal para aquellos trabajadores en sectores creativos, sabe 'vender' una imagen de ciudad estrechamente vinculada al acerbo cultural (Benach, 1993) y potencia desde las instancias públicas una determinada forma de hacer la 'ciudad creativa'.

\section{La especialización en la diversidad}

Desde una perspectiva meramente económica, Barcelona y su región metropolitana (RMB) siguen siendo una de las mayores regiones industriales de España. Sin embargo, no existe ningún sector que aporte de forma diferencial un alto porcentaje al PIB. Una de las características más rele-

4 En el distrito 22@, ejemplo claro de cambio de modelo económico por el que apuesta la ciudad, se han incorporado 1.502 empresas desde el año 2000 hasta 2009, suponiendo un aumento de 44.600 lugares de trabajo ("22@Barcelona: 10 anys de creixement econòmic" disponible en http://www.22barcelona.com/documentacio/22bcn_cens_2010.pdf). 
vantes del tejido industrial de Cataluña y, en particular de la RMB, es su falta de especialización y su composición basada en pequeñas y medianas empresas. Las consecuencias que ello supone tienen una doble vertiente: por un lado, la RMB se compone de un conglomerado policéntrico de ciudades con un liderazgo clave que es el de la ciudad de Barcelona (Trullén y Boix, 2003). Sinergias de complementariedad pueden identificarse, dedicándose principalmente la capital a la producción de servicios mientras que el resto de la región aún sigue especializado en la industria. Por otro lado, la tradición industrial proporciona elementos favorables para la producción flexible $\mathrm{y}$, sobretodo, para la posible transformación de sectores tradicionales como el textil o la edición de libros en nuevas industrias creativas como el diseño de moda o de juegos de ordenador (Pareja-Eastaway et al., 2007). Además, este entramado empresarial favorece soluciones de cooperación entre empresas más que de competencia, especialmente aquellas que basan su producción en la creatividad. La unicidad del talento y la especialización ayuda a generar lazos de cooperación y vínculos de integración entre empresas que, a la larga, favorecen el reparto del mercado de forma cooperativa y dialogada, facilitando así su adaptación a un entorno económico cambiante. Este es el caso, por ejemplo, del sector de producción de juegos de ordenador, software y publicaciones electrónicas, un sector con gran crecimiento en la región metropolitana de Barcelona, caracterizado por un sistema de producción "en red" en dónde la cooperación sustituye la competencia (Pareja-Eastaway et al., 2010).

Por otro lado, las empresas creativas en Barcelona se benefician indirectamente de la imagen que históricamente se ha labrado la ciudad como ciudad del diseño. Este hecho atrae a Barcelona a numerosos profesionales del sector que, de un modo u otro, además de partícipes directos del conglomerado creativo empresarial de la ciudad, encuentra en ella una fuente de inspiración.

\section{La histórica importancia de la cultura como motor de creatividad}

Ya en el siglo XIX, Barcelona se representa en círculos culturales como una de las vanguardias artísticas más relevantes en Europa (Cirlot, 1988). Este proceso no es ajeno al despegue económico de España desde principios del siglo XIX con Barcelona como uno de los motores industriales más importantes del país. La acumulación de riqueza propia del capitalismo propicia la aparición de una nueva burguesía con inquietudes culturales y artísticas sin la cual no hubiera sido posible el crecimiento y expansión de la ciudad a través de dos eventos culturales de escala internacional, las dos Exposiciones Universales de 1898 y 1929. En el primer caso, la ciudad regeneró el área portuaria y la antigua ciudadela mientras que en el segundo caso la ciudad recuperó la parte Sureste, sobretodo la montaña de Montjuic. La recuperación de la cultura catalana (iniciada ya con la 'Renaixença' en el siglo anterior, movimiento artístico que llega a las 
artes visuales como a la literatura) se convierte en leitmotiv de buena parte de las actividades de mecenazgo. Así pues, literatura, artes plásticas y arquitectura ${ }^{5}$ florecen paralelamente al crecimiento industrial de la ciudad.

La dictadura supone una ruptura completa con la tendencia iniciada en los años anteriores. Los movimientos vanguardistas dejan paso a tendencias más conservadoras y Madrid ejerce el papel de capital del país de modo centralizado, prescindiendo de otros centros urbanos como motores culturales. La cultura pues sostiene y refuerza el régimen sin ningún activo relevante reservado para Barcelona. Esta situación provoca el surgimiento de contracorrientes, mezcla de oposición política y reivindicación cultural, que supondrá para el futuro democrático una fuente de intelectualidad y nuevas aportaciones para la clase dirigente (Pareja-Eastaway et al., 2009).

Con la llegada de la democracia a final de los años setenta, Barcelona emerge como contraparte cultural y económica de Madrid. La recuperación del pasado cultural, la lucha por la cotidianeidad de la lengua catalana y el descubrimiento de nuevos retos para la ciudad configuran la hoja de ruta de los años posteriores. En particular, el uso de la cultura para dinamizar la ciudad no solamente en la esfera del ciudadano de "a pie" sino como polo de atracción en la escala internacional, se convierte en rasgo diferencial de la estrategia de largo plazo de Barcelona. En este contexto, la celebración de los Juegos Olímpicos en el año 1992 además de la consecución de un reto planteado en los años 80, afianza y vertebra los ejes de futuro de la ciudad.

La relevancia de la cultura y las industrias como pilar fundamental en la estrategia de futuro de la ciudad se constata a través de los sucesivos debates que se inician a partir de la implantación de los ayuntamientos democráticos en el país (Montaner, 2003). Aún siendo la Generalitat de Catalunya - gobierno autonómico - el último responsable de la política cultural, los gobiernos locales ejercen un papel decisivo en la promoción y estímulo de la cultura. En Barcelona, el uso y divulgación del acerbo cultural acumulado junto con la promoción de nuevas y creativas iniciativas vinculadas a la cultura desde los años noventa, supone uno de los mayores estímulos para visitar la ciudad, tanto de forma temporal, básicamente como turistas, como de forma más estable.

\section{Los factores soft de atracción de talento creativo y los factores network en Barcelona}

Barcelona ha mejorado, de forma objetiva, su posicionamiento internacional como ciudad de negocios. Este fenómeno no solamente se debe a la inversión real realizada en infraestructuras de transporte y nuevas tecnologías, factores

5 En este periodo, renombrados arquitectos catalanes - Gaudí, Domènech i Montaner i Puig i Cadafalch - desarrollan el llamado "Modernismo"; uno de los movimientos artísticos que hoy ejerce uno de los mayores atractivos para turistas y, de algún modo, inicia la tradición arquitectónica en la ciudad. 
tradicionales - factores hard - de atracción, sino también a la existencia de otros factores vinculados a la calidad de vida y al entorno en general - factores soft - que han permitido principalmente usar la imagen de una ciudad acogedora y tolerante como reclamo para atraer el talento creativo, especialmente el extranjero. La clásica distinción entre factores hard y soft en Barcelona se encuentra algo desdibujada ya que unos y otros se encuentran íntimamente conectados (Pareja-Eastaway et al., 2007).

Los factores soft son un elemento esencial en la atracción y retención de talento en Barcelona. Dos aspectos de los mismos deben tenerse en cuenta, por un lado, aquellos factores que son propios de la localización geográfica de la ciudad (clima, proximidad al mar, etc.), sin capacidad alguna para que sean modificados y, por otro, aquellos que son el resultado del histórico estímulo que han recibido las actividades culturales en un sentido amplio (Pareja-Eastaway et al., 2010).

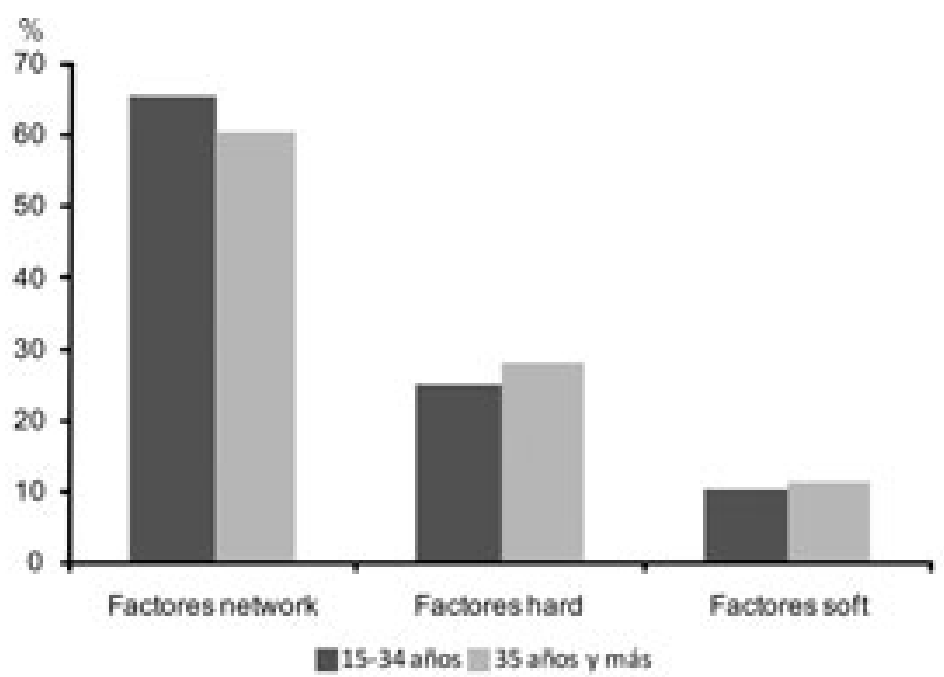

Fuente: Encuesta ACRE 2007

Fig. 3 - Factores que contribuyen a la satisfacción proporcionada por la RMB a trabajadores creativos y del conocimiento.

Fig. 3 -Determinants of the level of satisfaction with the Barcelona Metropolitan Area among creative and knowledge workers.

En Barcelona, el grado de satisfacción que manifiestan los trabajadores creativos y del conocimiento con la ciudad no dependen tanto de los aspectos que apuntan las hipótesis basadas en unos jóvenes creativos móviles a nivel global atendiendo al reclamo de las ciudades, buscando aquellos elementos que estimulan su talento y creatividad, como de los factores network o rela- 
cionales que para ellos representan los activos más valorados en relación a la satisfacción que proporciona trabajar y vivir en la ciudad, más allá del binomio hard-soft planteado habitualmente por la teoría (Florida, 2000). Entre estos se encuentran elementos conectados con sus trayectorias personales y su arraigo en el entorno (fig. 3).

Tal y como se puede apreciar en el gráfico, no existen grandes diferencias según la edad de los entrevistados. Son precisamente los factores que se revelan como determinantes los vinculados al haber nacido en la ciudad, tener familia o amigos viviendo ahí o el poder disfrutar de las redes sociales y profesionales que ofrece la ciudad de Barcelona. En cambio, los llamados factores soft - atmósfera, tolerancia, diversidad cultural etc. -, son, en términos relativos, los menos valorados.

Sin embargo, aquellos que proceden de otros países y se instalan en la ciudad de Barcelona para vivir y trabajar reconocen en ella un lugar ideal para vivir; la belleza de la ciudad, su dimensión humana, la amplia oferta de actividades de ocio y cultura, la herencia cultural del pasado - con la arquitectura como puntal - y la proximidad a zonas cercanas al mar y a la montaña son, entre otros, algunos de los aspectos más valorados (Pareja-Eastaway et al., 2010).

\section{LA CAPACIDAD DE BARCELONA PARA REINVENTARSE}

Uno de los componentes centrales en la competitividad urbana es la capacidad de transformación de las ciudades ante los nuevos retos económicos. En este sentido, en las postrimerías del siglo XX, las ciudades han tenido que hacer frente a una terciarización económica drástica que ha supuesto su transformación radical desde una especialización en producción industrial hasta ciudades de consumo. Sin embargo, la suerte que han corrido las ciudades en este proceso de transformación no ha sido el mismo: existen en cada ciudad elementos propios que favorecen una transformación, más o menos favorable, que a su vez les permite retener (y mejorar) su capacidad competitiva. En principio, parece razonable afirmar que aquellas ciudades con recursos inherentes vinculados a la creación de conocimiento se encontrarían en mejor punto de partida que aquellas con lagunas apreciables en estos aspectos y dificultades visibles en su proceso de adaptación al nuevo entorno (Crossa et al., 2010).

En cualquier caso, la reinvención de una ciudad se atribuye usualmente a la capacidad que la ciudad tiene para combatir problemas desarrollando nuevas trayectorias económicas, reorientando su especialización urbana, identificando objetivos de futuro, re-escribiendo el discurso oficial y consiguiendo renovar la confianza del ciudadano en la acción pública (Braithwaite y Levi, eds, 1998). Esta capacidad se encuentra íntimamente vinculada a las acciones asumidas por determinados actores $\mathrm{y}$, en especial, por las intervenciones públicas al respecto. 
La reinvención urbana en definitiva muestra mayoritariamente una aproximación top-down al futuro.

Barcelona y su región metropolitana son un ejemplo evidente de transición hacia la Nueva Economía: de una especialización altamente industrial se ha transformado en un territorio en el que la creatividad y el conocimiento son clave para el desarrollo económico. Existen ciertos aspectos que han favorecido esta transformación, en particular, la existencia de un tejido industrial previo diversificado que ha servido de base para la emergencia de una economía creativa y del conocimiento (Trullén, 2001). Sin embargo, el papel director y de liderazgo de las instancias públicas, principalmente el Ayuntamiento de Barcelona y privadas, sobretodo del Plan Estratégico de la Región Metropolitana de Barcelona, ha resultado esencial en el buen desarrollo del proceso.

\section{La promoción y el desarrollo urbano vinculado al evento}

Barcelona presenta una cierta tradición en la utilización de la celebración de eventos a escala internacional para beneficiarse de las sinergias positivas generadas al respecto y haciendo de ello una oportunidad para resolver 'temas pendientes' de la ciudad. Mientras que la Exposición Universal de 1888 consiguió rehabilitar y recuperar la zona próxima a puerto y a la vieja ciudadela, la Exposición de 1929 se propuso reconvertir los usos de la montaña de Montjuic. Del mismo modo, los Juegos Olímpicos de 1992 suponen el catalizador necesario para la mejora del transporte urbano a través de la construcción de las Rondes - vías de circunvalación alrededor de la ciudad - junto con la construcción de dos barrios de nueva creación, la Vila Olímpica de Horta y de Poblenou. La celebración del Fórum de las Culturas en el año 2004 permitió que la ciudad se dotara de nuevos equipamientos en una de las pocas áreas aún por urbanizar, fronteriza con Sant Andreu.

Aunque la celebración de eventos aporta una parte ciertamente visible en la renovación urbana de ciertas áreas, también se acompaña de un ideario que, tal y como se ha mencionado anteriormente, señala los elementos fundamentales en la definición de la 'receta'. Antes de la celebración de los Juegos Olímpicos, Barcelona necesitaba darse a conocer en el mundo: su acervo cultural, sus posibilidades de transformación institucional en un nuevo contexto democrático y su capacidad productiva eran, entre otros, los ejes de ésta estrategia. Para ello era necesario invertir grandes cantidades de dinero público para mejorar y desarrollar unas débiles y obsoletas infraestructuras que permitieran mejorar la conectividad. Los Juegos Olímpicos legitiman estas inversiones y en poco tiempo, Barcelona salta a la arena internacional de la competitividad urbana con un fuerte respaldo institucional que apoya a la ciudad a través de un perfecto ejercicio de venta de su imagen (Benach, 1993). Este fenómeno junto con la proliferación de vuelos low cost contribuye al asalto turístico masivo de la ciudad, generando tanto efectos negativos como positivos en Barcelona. 
Buena parte de los logros conseguidos durante las Olimpiadas (i.e. mejores infraestructuras, popularidad, dinamismo cultural) fueron utilizados más tarde como ejes en la estrategia de "vender" la ciudad al mundo. Otros acontecimientos que hicieran avanzar a la ciudad como la celebración del Fórum de las Culturas en el año 2004 quebrantó la simbiosis perfecta entre lo público, lo privado y lo colectivo que había caracterizado los Juegos Olímpicos Sin embargo, buena parte de la transformación del final de la Diagonal se debe a dicho evento. ${ }^{6}$

\section{La emergencia de una nueva gobernanza: más allá del modelo Barcelona}

La organización de los Juegos Olímpicos se basó en un liderazgo público fuerte y en un consenso colectivo alrededor del proyecto. El modelo Barcelona, basado en una eficiente gestión pública y en la utilización de la regeneración urbana como instrumento con un doble fin, tanto en su resolución de problemas urbanos pendientes como en la mejora del atractivo de la ciudad, se convirtió en ejemplo a seguir para otras ciudades (fig. 4).

El sistema de gobernanza resultante a raíz de la organización de los Juegos estaba basado en un liderazgo fuerte por parte municipal y en un consenso político puntual alrededor del proyecto. Es importante subrayar las circunstancias específicas que rodean el proceso y que atañen de forma única a la ciudad de Barcelona en ese momento; la democracia reciente, el entusiasmo de los primeros ayuntamientos democráticos, la participación ciudadana activa del momento y el papel crítico jugado por ciertos arquitectos son, entre otros, unicidades no transferibles del modelo. De ahí que la transferibilidad del modelo no siempre ha resultado posible o igualmente exitosa (Calvita y Ferrer, 2002).

La vigencia del Modelo en la actualidad ha sido y aún es, ampliamente discutida con defensores y detractores por igual (Montaner, 2003; Capel, 2005; Balibrea, 2006). Aspectos que en su día no fueron tenidos en cuenta como la proliferación del turismo de masas, la llegada repentina y masiva de la inmigración o retos de la sostenibilidad fuerzan a un replanteamiento del Modelo.

De los elementos que quedan como legado para la ciudad de ese Modelo, tres aspectos resultan particularmente interesantes para la Barcelona creativa del siglo XXI: el planeamiento estratégico, el fuerte liderazgo y la implicación de actores de la sociedad civil - asociaciones empresariales, sindicatos o agrupaciones de ciudadanos, son algunos ejemplos.

El planeamiento estratégico para la ciudad de Barcelona y su entorno metropolitano resulta uno de los puntos fuertes para su desarrollo a largo plazo. La definición de estrategias alrededor de objetivos consensuados a través del debate

6 Barcelona no cesa en el intento: en Enero de 2010 se ha anunciado su candidatura para la celebración de los Juegos Olímpicos de invierno en el año 2022. 
y la discusión implicando tanto a agentes públicos como privados ha permitido el establecimiento de prioridades previamente negociadas. Así, la orientación de la ciudad hacia la economía del conocimiento o la creatividad no es producto de la casualidad sino de apuestas escogidas a priori.

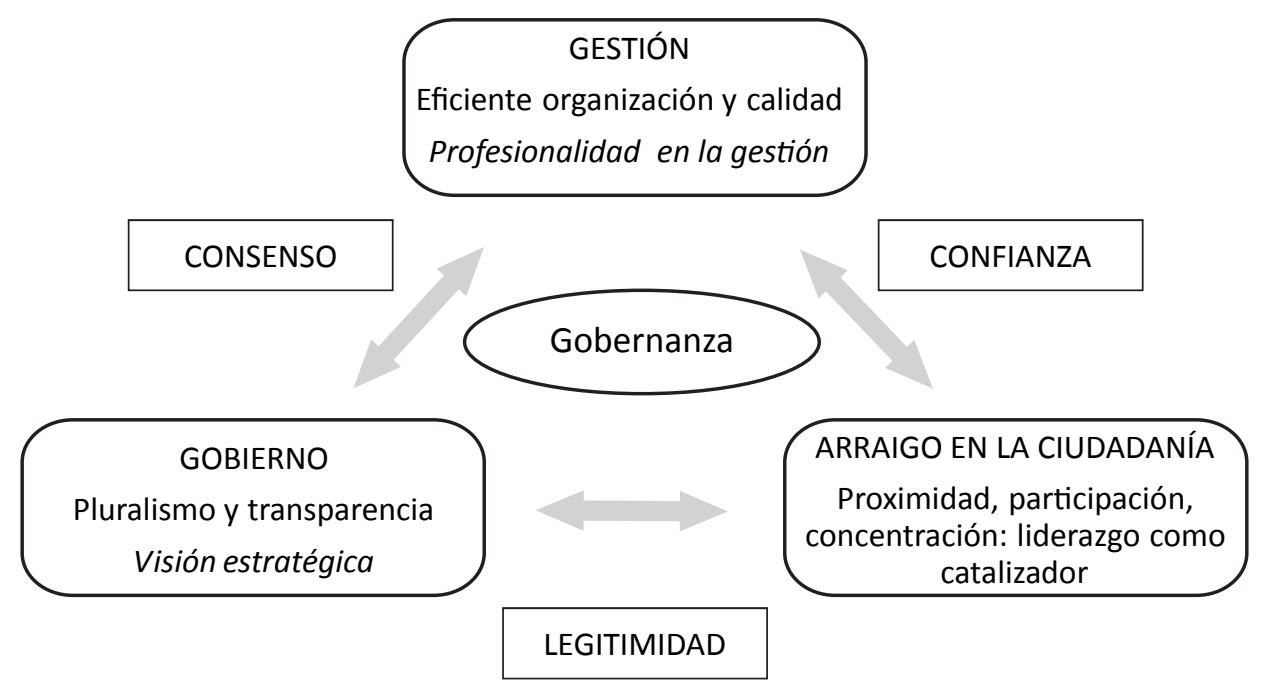

Fuente: Basado en Monclús, 2003.

Fig. 4 - Síntesis del Modelo Barcelona.

Fig. 4 - Summary of the Barcelona Model.

La inclusión de otros actores más allá del sector público en todas sus instancias ha sido también una de las herencias diferenciales del Modelo Barcelona? Los modelos de parteneriado público-privado han sido referente para nuevos proyectos como por ejemplo, la renovación del frente marítimo Norte y, en particular, la creación del nuevo distrito digital del 22@ (Pallarès-Barbera y Casellas, 2009). En ellos es indiscutible el fuerte papel de liderazgo ejercido por el Ayuntamiento de Barcelona. Uno de los puntos fuertes a añadir resulta la capacidad de adaptar el papel de líder a las circunstancias cambiantes tanto del entorno como del marco económico global. La flexibilidad en la ejecución y en la integración de nuevos elementos a lo largo de los proyectos se convierte en un factor diferencial de otros papeles de liderazgo (Barber y Pareja-Eastaway, 2010).

7 Del total de inversión que supusieron los Juegos Olímpicos, alrededor de 8.000 millones de euros, un $32,7 \%$ fue financiado por empresas privadas y el resto $(67,3 \%)$ por el sector público (Estado, 33,9\%; Generalitat de Catalunya, 15\%; Ayuntamiento, 10,5\%). 


\section{LA ESTRATEGIA DE APOYO A LA CREATIVIDAD Y LA CULTURA}

La Agenda de Lisboa (2000) claramente identifica el desarrollo de la economía del conocimiento y la creatividad como máximo objetivo para el año 2010: "convirtiéndose la UE en la economía mundial más competitiva y dinámica basada en el conocimiento, capaz de generar un crecimiento sostenible, con más y mejores lugares de trabajo y una mayor cohesión social" (Agenda de Lisboa, 2000: 3).

El sector cultural es uno de los ejes centrales de la agenda local de la ciudad de Barcelona vinculado a la planificación estratégica (Manito, 2008). Desde las instancias municipales de la ciudad de Barcelona existe el convencimiento de que las políticas creativas benefician no únicamente a las industrias creativas sino al total de la estrategia competitiva de la ciudad, Barcelona se considera como producto cultural en sí mismo siendo cultura y creatividad uno de los ejes fundamentales de influencia en el panorama urbano, económico y social (Informe COMPETE, 2008).

Barcelona apuesta por la cultura y los sectores creativos como vía de acceso a la economía del conocimiento, sin menospreciar su capacidad de generación de valor añadido y dinámica económica, reconociendo asimismo su papel de motor de desarrollo, por encima de otros sectores económicos. En este sentido, son muchos los esfuerzos para conseguir remediar uno de los puntos débiles del sector, esto es, identificar su dimensión precisa y contribución real al crecimiento económico de Barcelona en particular y de Catalunya en general. En el año 1999, ve la luz el Llibre Blanc de la Cultura a Catalunya elaborado por la Generalitat de Catalunya, antecesor del Llibre Blanc de les Indústries Culturals a Catalunya publicado el año 2003, bajo la dirección de Lluis Bonet y como consecuencia inmediata de la creación del Institut de les Indústries Culturals de Catalunya el año 2002. Posteriormente, la Cámara de Comercio, Industria y Navegación de Barcelona presenta en el 2005 un estudio completo sobre el sector El sector cultural a Catalunya. Múltiples indicadores se elaboran en el año 2006 con el objeto de conocer en profundidad las principales magnitudes y estadísticas de la economía de la cultura en Catalunya, Indicadors i estadístiques culturals a Catalunya, culminando en la primera revisión del Plan Estratégico de la Cultura para la ciudad de Barcelona en ese mismo año.

Todas las reflexiones que surgen de estos documentos constatan la importancia del sector cultural como base para el desarrollo de diversas actividades creativas así como la necesidad de mejorar y reforzar los ejes de su competitividad. Además, uno de los elementos a subrayar es la importancia del contexto empresarial y de calidad de vida en el qué se desarrollan las industrias culturales en Barcelona, y ello especialmente por su capacidad de atracción de inversión extranjera hacia la ciudad.

La necesidad de reforzar el tejido industrial creativo "a través del diálogo, nuevos fondos, y ayuda renovada a la exportación de productos culturales y asegurar la presencia en el mercado nacional" (Bonet, 2002) se manifiesta tanto 
a instancias de Comunidad Autónoma ${ }^{8}$ como de Ayuntamiento. En particular, la estrategia de apoyo a las industrias creativas llevada a cabo por el Ayuntamiento de Barcelona se basa en cinco pilares (Informe COMPETE, 2008):

Incrementar el valor añadido creativo a aquellas industrias tradicionales que reflejan un elevado componente cultural y económico pero que ocupan un segundo lugar en cuanto a peso específico en la ciudad.

Cooperar con el sector privado a través de iniciativas en las que los esfuerzos conjuntos representen un mutuo beneficio.

Facilitar la conexión entre las diferentes realidades culturales, iniciativas y empresas creativas de la ciudad.

Compartir el riesgo con el sector privado a través de apoyo directo o ideando nuevos instrumentos financieros (i.e. fondos de capital riesgo).

Internacionalizar la ciudad a través de la atracción de profesionales creativos.

Otros estudios (Pareja-Eastaway et al., 2009) señalan la relevancia de la educación como motor de cambio, que atrae y crea talento, generando el capital humano necesario para el desarrollo económico próspero de la ciudad.

Tal y como señala Mascarell (2006), las políticas públicas en Barcelona "otorgan un papel central a personas, entidades, empresas proyectos públicos y centros educativos que generen contenidos, ideas, servicios y productos innovadores y de alto valor añadido" (Mascarell, 2006: 5). La ciudad debería reforzar el potencial de las industrias creativas y culturales como contribuidoras al crecimiento económico, intensificando la relación entre los sectores públicos y privados, fomentando las iniciativas que favorezcan el surgimiento de la creatividad (fig. 5). Todo ello sin menoscabo de la importancia de integrar a la ciudadanía (incluyendo a la población inmigrada) en este proyecto de ciudad, dibujando una cultura cívica compartida.

Desde el año 2008, el Ayuntamiento de Barcelona ayuda a la reconversión de espacios públicos de tradición fabril en laboratorios de creación artística. Esta estrategia aúna el esfuerzo de recuperar el pasado histórico industrial con la voluntad de estimular la capacidad creadora de los artistas en Barcelona.

La gestión de estos espacios es diversa, existen "fábricas" con una autogestión por parte de aquellos que utilizan el espacio (i.e Hangar - http://www.hangar. org/drupal/) así como ejemplos en que la gestión se mantiene en manos del Ayuntamiento (i.e. Fabra i Coats).

Esta experiencia innovadora ha sido también puesta en marcha por otras ciudades como Ámsterdam, Berlín o Marsella. Este tipo de espacio altamente flexible facilita todo tipo de transformaciones adecuándose sin excesivo esfuerzo a múltiples usos.

8 En el Año 2000 se crea el Institut de les Indústries Culturals en el seno del Departament de Cultura de la Generalitat de Catalunya. 
El apoyo institucional a esta iniciativa permite crear las condiciones oportunas para superar las dificultades que entraña el alto crecimiento del precio del suelo, tanto para aquellos que quieren acceder a su uso como para aquellos que se plantean su conversión en otro tipo de uso mucho más rentable como el residencial.

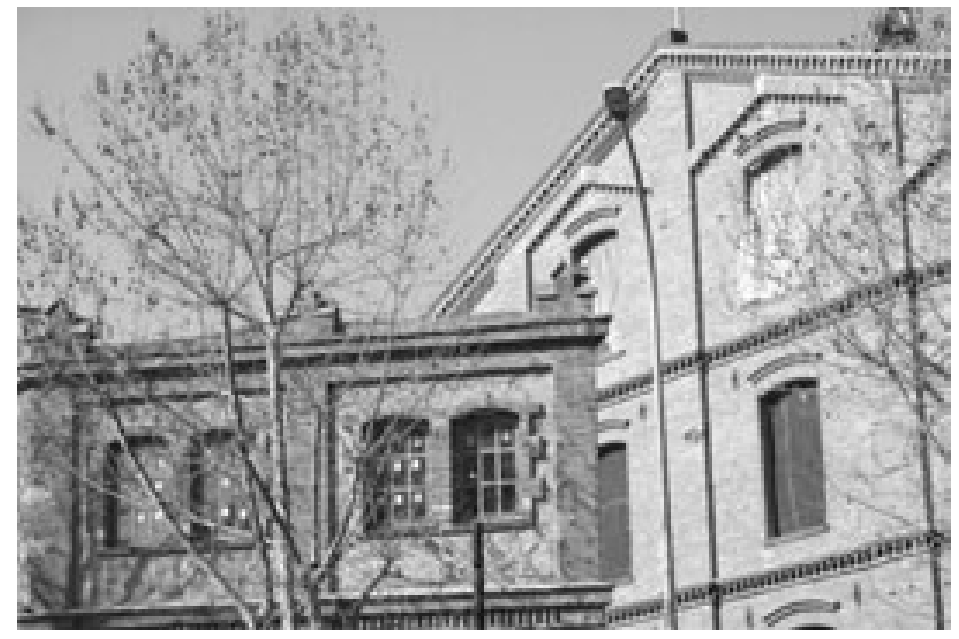

Fig. 5 - Fábricas para la creación (Fàbriques per a la creació). Fig. 5 - Creative factories.

\section{CONCLUSIONES}

Barcelona es una ciudad creativa. No solo lo dicen aquellos que viven y trabajan en ella sino también los que forman parte del entramado público y privado que conforma su sociedad civil.

Este artículo se plantea la identificación de los condicionantes o pre-requisitos para considerar a Barcelona como ciudad 'creativa'. Tanto el sustrato económico, social y cultural de la ciudad como la iniciativa liderada por las instancias públicas suponen uno de los factores diferenciales propios de la ciudad. La especialización flexible en la diversidad de las empresas de la RMB favorece la transformación de los sistemas de producción tradicionales en aquellos que incorporan la creatividad como factor esencial en la cadena de valor. Las formas de creación cultural y las industrias culturales tienen un arraigo histórico en la ciudad y proporcionan parte de los elementos que contribuyen al atractivo de la ciudad. De ese modo, Barcelona muestra una presencia elevada de los factores de localización soft que representan una de las razones por las que muchos trabajadores creativos desean vivir y trabajar en la ciudad. Sin 
embargo, son los factores network vinculados a las trayectorias personales y profesionales de los trabajadores creativos el elemento más importante que explica la presencia de los mismos en la ciudad.

Una de las características particulares de la ciudad es su capacidad para reinventarse ante la necesidad de adecuarse al contexto económico cambiante y la presencia de nuevas realidades como la inmigración o la exigencia de un desarrollo sostenible. La iniciativa local no es ajena a este proceso; las instancias públicas en Barcelona han utilizado parte del acerbo cultural para la creación de una determinada imagen de ciudad que ha favorecido sin duda a buena parte de las actividades económicas instaladas en la ciudad. Además, el planteamiento estratégico de largo plazo ha posibilitado la definición de objetivos y especializaciones estables de crecimiento. El liderazgo del municipio ha sido determinante así como su capacidad para adaptarse a las necesidades de los nuevos actores creativos presentes en la ciudad.

Sin embargo, algunos de los retos conseguidos - poner a la ciudad en el ranking de las ciudades europeas - han generado efectos externos no deseados (i.e. la presencia de turismo masivo) que no ayudan a la identificación del imaginario colectivo de aquellos que viven en Barcelona con la voluntad municipal. La apuesta por la atracción de un determinado tipo de conocimiento, creativo y altamente cualificado debe complementarse con elementos de intervención pública que reafirmen el objetivo de la cohesión social.

\section{AGRADECIMIENTOS}

Este artículo has sido posible gracias a mi participación en el proyecto ACRE (Accommodating Creative Knowledge - Competitiveness of European Metropolitan Regions within the Enlarged Union) financiado por el $6^{\circ}$ Programa Marco de la UE y al trabajo del resto de miembros del equipo ACRE en Barcelona.

\section{BIBLIOGRAFÍA}

Amin A (ed) (1994) Post-fordism, a reader. Balckwell, London.

Aurigi A (2005) Making the digital city. The early shaping of urban internet space. Ahsgate, Aldershot.

Balibrea M P (2006) Barcelona: del modelo a la marca. En Forum de cultura, democratizem la democràcia. [Acedido a 19 noviembre 2006] http://www.e-barcelona.org

Benach N (1993) Producción de imagen en la Barcelona del 92. Estudios Geográficos, LIV(212): 453-504.

Bonet L (2002) Llibre Blanc de les Indústries Culturals de Catalunya. Barcelona

Braithwaite V, y Levi M (eds) (1998) Trust and governance. Russell Sage Foundation.

Capel H (2005) El modelo Barcelona: un examen crítico. Ediciones del Serbal.

Cirlot L (1988) Las claves de las vanguardias artísticas en el siglo XX. Editorial Ariel, Barcleona.

Clusa J (1999) La experiencia urbanística de Barcelona, 1986-1992 y las expectativas del Forum 2004. Ciudades, 5: 85-102

Crossa V, Pareja-Eastaway M, Barber A (2010, forthcoming) Reinventing the city: Barcelona, Birmingham and Dublin. In Musterd S, Murie A (eds) Making competitive cities. Blackwell-Wiley, London. 
Florida R (2002) The rise of the creative class. Basic Books, New York.

Generalitat de Catalunya, Departament de Cultura (2006) Indicadors i estadistiques culturals a Catalunya. http://www20.gencat.cat/docs/CulturaDepartament/Cultura/Documents/Arxiu/ Arxius\%20GT/Indicadors_estadistiques_culturals_Catalunya.pdf

Informe COMPETE, (2008) The COMPETE Network: Final Report. Messages for Competitive European Cities. http://www.ljmu.ac.uk/EIUA/EIUA_Docs/COMPETE_Final_Report.pdf

Inneraritty D (2007) Gobernar los nuevos espacios: entre lo local y lo global. Ponencia. 3er Seminari Pla Estratègic Metropolità de Barcelona.

Landry C (2000) The creative city. A toolkit for urban innovators. Earthscan, London.

Manito F (2008) Cultura $i$ estrategia de ciutat. La centralitat del sector cultural a l'agenda local. Collecció Obres Digitals. Escola de 1'Administració Pública de Catalunya.

Marcuse P, van Kempen R (eds.) (2000) Globalizing cities. A new spatial order? Blackwell, Oxford.

Mascarell F (2006) Polítiques creatives a Barcelona. COMPETE network conference, Barcelona.

Monclús F X (2003) The Barcelona model. An original formula? From reconstruction to Strategic Urban Projects (1979-2004). Planning Perspectives, 18(4): 399-421.

Montaner J M (2003) Repensar Barcelona. Edicions UPC, Barcelona.

Musterd S, Murie A (2010, forthcoming) Making creative cities. Wiley-Blackwell, Hoboken, USA.

Observatori Barcelona (2008) Informe 2008. Ajuntament de Barcelona i Cámara de Comerç de Barcelona.

Pallares-Barbera M y Casellas A (2009) Public-sector intervention in embodying the new economy in inner urban areas: the Barcelona experience. Urban Studies, 46: 1137-1155.

Pareja-Eastaway M, Turmo Garuz J, García Ferrando L, Pradel i Miquel M (2010) Approaching the Barcelona Metropolitan Region. The views of high-skilled employees, managers and high qualified immigrants. ACRE report 8.2.

Pareja-Eastaway M, Turmo Garuz J, García Ferrando L, Pradel i Miquel M (2009) The magnetism of Barcelona. The view of transnational migrants. ACRE report 7.2.

Pareja-Eastaway M, Turmo Garuz J, Pradel i Miquel M, García Ferrando L, Simó M (2007) The city of marvels? Multiple endeavours towards competitiveness in Barcelona. ACRE report 2.2.

Sassen S (1991) The global city. New York, London, Tokyo. Princeton University Press, Princeton, NJ.

Scott A J (2006) Social economy of the metropolis. Cognitive-cultural capitalism and the global resurgence of cities. Oxford Unviersity Press, Oxford.

Trullén J (2001) El proyecto Barcelona - ciudad del conocimiento desde la economía. In Moscono F, Solé Parellada F, Chantiri A (Eds.) Política industrial y tecnológica II. Edicions de la Universitat Politécnica de Catalunya, Barcelona: 111-124.

Trullén J, Boix R (2003) Barcelona, metrópolis policéntrica en red. Cerdanyola del Vallès: Departament d'Economia Aplicada, Universitat Autònoma de Barcelona. 